\title{
High MMP-9 activity characterises pleural tuberculosis correlating with granuloma formation
}

\author{
P. Sheen*,\#, C.M. O’Kane", K. Chaudhary ${ }^{+}$, M. Tovar*, C. Santillan*, J. Sosa ${ }^{\S}$, \\ L. Caviedes*, R.H. Gilman*,*, G. Stamp ${ }^{+}$and J.S. Friedland
}

ABSTRACT: Tuberculosis (TB) pleural disease is complicated by extensive tissue destruction. Matrix metalloproteinase (MMP)-1 and -9 are implicated in immunopathology of pulmonary and central nervous system TB. There are few data on MMP activity in TB pleurisy. The present study investigated MMP-1, -2 and -9 and their specific inhibitors (tissue inhibitor of metalloproteinase (TIMP)-1 and -2) in tuberculous effusions, and correlated these with clinical and histopathological features.

Clinical data, routine blood tests, and pleural fluid/biopsy material were obtained from 89 patients presenting with pleural effusions in a TB-endemic area. MMP-1, -2 and -9 were measured by zymography or western blot, and TIMP-1 and -2 by ELISA. Pleural biopsies were examined microscopically, cultured for acid-alcohol fast bacilli and immunostained for MMP-9.

Tuberculous pleural effusions contained the highest concentrations of MMP-9 compared with malignant effusions or heart failure transudates. MMP-9 concentrations were highest in effusions from patients with granulomatous biopsies: median (interquartile range) $108(61-218) \mathrm{pg} \cdot \mathrm{mL}^{-1}$ versus $43(12-83) \mathrm{pg} \cdot \mathrm{mL}^{-1}$ in those with nongranulomatous pleural biopsies. MMP-1 and -2 were not upregulated in tuberculous pleural fluid. The ratio of MMP-9:TIMP-1 was significantly higher in TB effusions.

Tuberculous pleurisy is characterised by a specific pattern of matrix metalloproteinase-9 upregulation, correlating with the presence of granulomas and suggesting a specific role for matrix metalloproteinase-9 in inflammatory responses in tuberculous pleural disease.

KEYWORDS: Extracellular matrix, human, inflammation, matrix metalloproteinases, pleura, tuberculosis

$\mathbf{T}$ uberculosis (TB) remains a leading global health burden, with more than 8 million new cases and approximately 2 million deaths per year. Pleural involvement can occur as a manifestation of primary TB where the Ghon focus ruptures into the pleural cavity, or as a complication of reactivation TB. Pleural involvement is more common in primary disease in high incidence areas by a factor of approximately four, and in younger patients [1]. Tuberculous pleural effusions are characterised by an intense inflammatory component with mononuclear cells [2]. The inflammatory response causes disruption of the normal pleural cavity tissue, which may restrict normal lung expansion even after successful anti-tuberculous therapy. The mechanisms by which tissue damage is induced in tuberculous pleural disease remain unclear, and the role of broad-spectrum anti-inflammatory therapy (in the form of corticosteroid treatment) is unclear.
The matrix metalloproteinases (MMPs) are potentially important mediators of inflammatory responses and tissue destruction in TB. The MMPs form a group of zinc-dependent proteases capable of degrading all components of the extracellular matrix [3]. They are upregulated in many inflammatory conditions, where they contribute to leukocyte recruitment by facilitating matrix breakdown. In addition, MMPs may regulate cytokine responses, for example MMP-2, -3 and -9 can activate interleukin (IL)$1 \beta$, promoting inflammation [4]. In contrast, MMP-2-mediated cleavage of monocyte chemotactic protein-3 may dampen the inflammatory response [5]. Excessive MMP secretion has been linked with tissue damage in rheumatoid arthritive pulmonary disease $[7,8]$. Recently, increased MMP-9 (gelatinase B) activity has been identified in animal models [9] and human TB [10-12]. tis [6], pulmonary fibrosis and chronic obstruc-

\section{AFFILATIONS}

*Dept of Microbiology, Universidad Peruana Cayetano Heredia.

${ }^{\S}$ Dept of Pulmonology, Dos de Mayo Hospital, Lima, Peru.

\# Dept of International Health, Johns Hopkins University, School of

Hygiene and Public Health, Baltimore, MD, USA.

'Dept of Infectious Diseases and Wellcome Trust Centre for Clinical Tropical Medicine, and

${ }^{+}$Dept of Pathology, Imperial College London, Hammersmith Campus,

London, UK.

\section{CORRESPONDENCE}

J.S. Friedland

Dept of Infectious Diseases and Immunity

Faculty of Medicine

Imperial College

Du Cane Rd

Hammersmith Hospital

London

W12 ONN

UK

Fax: 442083833394

E-mail: j.friedland@imperial.ac.uk

Received:

September 272007

Accepted after revision:

July 302008

\section{SUPPORT STATEMENT}

The authors are grateful for support from the National Institute for Health Research Biomedical Research Centre funding scheme at Imperial College (London, UK).

STATEMENT OF INTEREST

None declared.

European Respiratory Journal

Print ISSN 0903-1936

Online ISSN 1399-3003 
Elevated MMP-9 concentrations were found in cerebrospinal fluid (CSF) and brain tissue in tuberculous meningitis, which correlated with local neuropathology and death [10,13].

The MMPs are classified functionally according to their relative substrate specificity. For example, collagenases (MMP-1, -8 and -13) degrade type I collagen whereas gelatinases (MMP-2 and -9) degrade gelatin (denatured collagen) and type IV collagen, a major component of basement membranes. However, this classification is artificial, as there is overlap in substrate specificity and redundancy of function among MMPs [14]. MMP secretion is tightly regulated by several mechanisms, including transcriptional regulation, compartmentalisation and secretion of pro-forms that require activation. A further key regulator is concomitant secretion of tissue inhibitors of metalloproteinases (TIMPs) by the cells that secrete MMPs. TIMPs bind with MMPs in a 1:1 stoichiometric ratio, such that in general the matrix-degrading capacity is determined by the ratio of MMP to TIMP secretion.

MMPs have been identified in pleural effusions of inflammatory and malignant aetiology [15]. There are few data on MMP expression in tuberculous pleural effusions. Two studies have shown upregulation of several MMPs measured by ELISA (including MMP-1, -2, -8 and -9) in tuberculous pleural fluid compared with transudates but have not related these to clinical data or correlated the MMP expression pattern with histological findings [16, 17]. In a further study, PARK et al. [18] demonstrated upregulated MMP-9 in tuberculous effusions compared with cancer and heart failure, but did not measure other MMPs or TIMPs that may be functionally important in the pleural space. Tuberculous pleural effusions are characterised by high monocyte and lymphocyte counts. MMP-9 is quantitatively the most important MMP secreted by monocytes and macrophages, and may also be secreted by activated lymphocytes [19]. Infection of monocytes and macrophages with Mycobacterium tuberculosis in vitro and in vivo has been shown to induce MMP-9 secretion [11, 12]. MMP-9 secretion is of particular interest in pleural disease, since, as a gelatinase, it degrades type IV collagen and may contribute to loss of integrity of the basement membrane around blood vessels and under the mesothelial layer, leading to fluid accumulation in the pleural space.

Monocytes and macrophages may also secrete high MMP-1 concentrations. In addition, parenchymal cells of the pleural space, such as fibroblasts and mesothelial cells, secrete MMP-1 (interstitial collagenase) constitutively [20] and in response to inflammatory cytokines secreted by TB-infected monocytes and macrophages [21]. Proteases such as tissue plasminogen activator and plasmin, which are present in the pleural space [22], may activate MMPs secreted by inflammatory leucocytes and parenchymal cells and perpetuate a cycle of connective tissue breakdown and further inflammatory cell recruitment. The present study was designed to investigate whether MMP-1 and -9 and their specific inhibitors, TIMP-1 and -2 , have a role in the pathogenesis of pleural TB. Elucidating the contribution of MMPs to tissue damage and disease in tuberculous pleurisy may provide a potential therapeutic target to reduce the longterm sequelae of pleural adhesions and pleural rind formation. Targeted MMP inhibition is being developed for cancer [23] and may become a therapeutic option in inflammatory diseases [24], while broad-spectrum MMP inhibition can be achieved by simple drugs such as tetracyclines [25].

\section{METHODS}

\section{Study subjects}

All subjects were adults admitted to the Respiratory Service at Dos de Mayo Hospital (Lima, Peru) over a 6-month period. Demographic data, including age, sex and mass, were collected for each patient; clinical symptoms and purified protein derivative (PPD) reaction (where available) were also recorded. Patients who had been symptomatic for $>2$ weeks, with chest radiograph evidence of pleural effusion, underwent diagnostic thoracocentesis, with pleural biopsy if clinically indicated. In total, 80 patients underwent pleural biopsy using an Abrams needle under sterile conditions and taking one to eight samples per patient. As part of the routine diagnostic work-up, the following investigations were carried out on pleural fluid: biochemistry (total protein, albumin and adenosine deaminase), cytological examination and differential leukocyte count, and microbial culture, including culture for M. tuberculosis. Pleural biopsies were sent for culture and histopathological examination. Routine haematoxylin/eosin and auramine staining were performed.

\section{Stratification}

Patients were stratified into three main groups: definite pleural TB (defined as a positive mycobacterial culture of pleural biopsy/fluid or granuloma on pleural biopsy with or without caseation or positive PCR); probable pleural TB (defined as the presence of clinical or other laboratory features suggestive of $\mathrm{TB}$, but with culture-negative pleural fluid or biopsy); and negative controls (pleural effusion due to congestive cardiac failure). A further group of 10 patients with pleural effusions due to metastatic carcinoma served as a positive control for the analysis of MMP expression. Pleural effusions due to metastatic carcinoma are associated with high MMP-2 and -9 expression [26]. Ethical approval for the study was from the Institutional Review Boards of Johns Hopkins School of Public Health (Baltimore, MD, USA) and A.B. Prisma (Lima, Peru).

\section{Processing of pleural fluid for MMP and TIMP measurements}

Pleural fluid was centrifuged at 2,000 $\times g$ for $5 \mathrm{~min}$ and cellfree supernatant stored at $-70^{\circ} \mathrm{C}$ for later analysis. MMP-1 and -9 and TIMP-1 and -2 were found to be stable over repeated freeze-thaw cycles. All measurements were carried out with the operator blinded to clinical data.

\section{Zymography}

The concentrations of MMP-2 and -9 in pleural fluid were measured by gelatin zymography as previously described [12].

\section{Western blotting}

Western blotting was performed to confirm the identity of bands obtained by gelatin zymography as MMP-9, and to measure MMP-1 concentrations. Equal quantities of protein (as determined by Bradford assay) were heat-inactivated at $100^{\circ} \mathrm{C}$ for $2 \mathrm{~min}$ and resolved on $10 \%$ acrylamide gels. Protein was electrically transferred onto a nitrocellulose membrane, which was blocked with either 5\% bovine serum albumin for MMP-9 detection, or 20\% milk protein for MMP-1 detection, for $1 \mathrm{~h}$. 
Incubation with primary antibody (either MMP-1 or -9 , both from The Binding Site (Birmingham, UK) at 1/1000 dilution) was carried out overnight before incubation with secondary antibody (peroxidase-conjugated anti-sheep immunoglobulin (Ig)G at 1/1000 dilution; The Binding Site). Bound antibody was visualised by chemiluminescence and exposure of the membrane to light-sensitive film. The optical density of the bands from pleural fluid samples was measured using Scion image analysis and compared for quantification with that from the known standard aliquot of MMP-1 or -9 .

\section{TIMP-1 and -2 measurements}

TIMP-1 and -2 concentrations in pleural fluid were measured by ELISA (BioSource ${ }^{\mathrm{TM}}$ CytoSet $^{\mathrm{TM}}$ TIMP-1 ELISA (Invitrogen, Paisley, UK) and DuoSet ${ }^{\circ}$ TIMP-2 ELISA (R\&D Systems Europe Ltd, Abingdon, UK) developed according to the manufacturers' instructions. The lower limits of detection of the TIMP-1 and TIMP-2 ELISAs were $1.6 \mathrm{ng} \cdot \mathrm{mL}^{-1}$ and $30 \mathrm{pg} \cdot \mathrm{mL}^{-1}$, respectively.

\section{Immunohistochemistry for MMP-9 and TIMP-1}

Pleural biopsies were examined for MMP-9 and TIMP-1 expression using the 3,3'- diaminobenzidine (DAB) system. Briefly, sections were dewaxed and then treated with $0.6 \%$ hydrogen peroxide to deplete endogenous peroxidase activity. Sections for TIMP-1 immunostaining were boiled in $10 \mathrm{mM}$ citrate buffer ( $\mathrm{pH}$ 6.0) followed by cooling at room temperature for $20 \mathrm{~min}$. Sections for MMP-9 staining did not require boiling in citrate. Sections were then blocked with 5\% normal goat serum and, after washing in PBS, incubated at room temperature with either mouse monoclonal anti-human MMP-9 antibody (1/25 dilution) or mouse monoclonal antihuman TIMP-1 antibody (1/50 dilution; NeoMarkers, Fremont, CA, USA) for $2 \mathrm{~h}$. After washing in PBS-Tween buffer, biotinylated goat anti-mouse IgG (1/500 dilution) was added, followed by peroxidase-labelled streptavidin. DAB chromogen was applied for $10 \mathrm{~min}$ and slides counterstained with haematoxylin. As positive controls, placental tissue was stained for MMP-9 and breast carcinoma for TIMP-1. Negative controls for the antibodies used have been previously published [12]. Samples were analysed by two pathologists blinded to the nature of the specimens and the results of microbiology.

\section{Statistical analysis}

Comparisons between groups were made using the Fisher's exact test for categorical demographic and clinical data, one-way ANOVA for normally distributed variables, and the KruskallWallis H- or Mann-Whitney U-test for nonparametric data. A $\mathrm{p}$-value $<0.05$ was taken as significant.

\section{RESULTS}

Pleural fluid was sampled from 89 patients, of whom 74 were studied in more detail: 49 patients with a diagnosis of pleural $\mathrm{TB}$, nine patients with probable pleural $\mathrm{TB}$, six control patients with congestive cardiac failure and 10 positive control patients with metastatic malignant pleural effusions (confirmed by the presence of mitotic cells in the pleural fluid or on pleural biopsy). The remaining patients had a final diagnosis of cirrhosis $(n=2)$, nephrotic syndrome $(n=1)$, rheumatoid arthritis $(n=1)$ or "unknown aetiology" $(n=11)$, and were not included in further analysis. Of the patients in the "pleural TB" group, all 49 patients underwent pleural biopsy; in the "probable pleural $\mathrm{TB}^{\prime}$ group, four out of nine underwent biopsy; in the "carcinoma" group, nine out of 10 underwent biopsy; and in the "heart failure" group, pleural biopsies were carried out in one out of the six patients.

Of the 49 patients with pleural TB, 42 had a positive culture on pleural fluid or pleural biopsy culture (40 positive biopsy cultures and 11 positive pleural fluid cultures). Seven patients were culture negative, but all had granulomatous change on pleural biopsy, with three showing caseating granulomas. Two of the cases had positive PCR tests (for TB) on pleural biopsy, of which one had mycobacteria on auramine staining of the biopsy specimen. Granulomas were not detected in biopsy material from any of the patients with carcinoma or heart failure.

The probable pleural TB patients had clinical history compatible with $\mathrm{TB}$, mononuclear pleural effusions with high

TABLE 1 Summary of histological and culture findings in pleural biopsy and fluid

\begin{tabular}{|c|c|c|c|c|}
\hline & Heart failure & Pleural TB & Probable pleural TB & Carcinoma \\
\hline Biopsy attempted & 1 & 49 & 4 & 9 \\
\hline Pleural tissue obtained & 1 & 47 & 2 & 8 \\
\hline Granulomas on pleural biopsy & 0 & 40 & 0 & 0 \\
\hline Pleural biopsy & 0 & 3 & 0 & 0 \\
\hline Pleural fluid & 0 & 0 & 0 & 0 \\
\hline \multicolumn{5}{|l|}{ Positive culture } \\
\hline Pleural biopsy & 0 & 40 & 0 & 0 \\
\hline Pleural fluid & 0 & 11 & 0 & 0 \\
\hline
\end{tabular}




\begin{tabular}{|c|c|c|c|c|}
\hline \multirow[t]{2}{*}{ TABLE 2} & \multicolumn{4}{|c|}{$\begin{array}{l}\text { Clinical features of patients and controls at } \\
\text { recruitment }\end{array}$} \\
\hline & $\begin{array}{l}\text { Heart } \\
\text { failure }\end{array}$ & $\begin{array}{c}\text { Pleural } \\
\text { TB }\end{array}$ & $\begin{array}{l}\text { Probable } \\
\text { pleural TB }\end{array}$ & Carcinoma \\
\hline Patients n & 6 & 49 & 9 & 10 \\
\hline Age yrs & $68.5 \pm 6.8$ & $35.1 \pm 2.7^{\star}$ & $32.7 \pm 4.4^{*}$ & $65.8 \pm 5.0$ \\
\hline BMI $\mathbf{k g} \cdot \mathrm{m}^{-2}$ & $20.4 \pm 2.2$ & $22.7 \pm 0.6$ & $21.8 \pm 1.30$ & $23.40 \pm 1.95$ \\
\hline Pyrexia & 16.7 & $65.3^{\#}$ & $88.9^{\#}$ & 50.0 \\
\hline Weight loss & 50.0 & 81.6 & 77.8 & $90.0^{\#}$ \\
\hline Night sweats & 33.3 & 49.0 & 33.3 & 20.0 \\
\hline Dyspnoea & 83.3 & 88.9 & 83.7 & 100.0 \\
\hline Pleuritic pain & 33.3 & $83.7^{\#}$ & $100^{\#}$ & 70.0 \\
\hline Cough & 66.7 & 69.4 & 55.5 & 70.0 \\
\hline Haemoptysis & 0 & 4.08 & 0 & 10.0 \\
\hline Positive PPD tes & 33.3 & $83.8^{\#, \cdot}$ & 57.1 & 25.0 \\
\hline \multicolumn{5}{|c|}{$\begin{array}{l}\text { Data are presented as mean } \pm \text { SEM or } \% \text { of patients, unless otherwise stated. TB: } \\
\text { tuberculosis; BMI: body mass index; PPD: purified protein derivative. *: } p<0.05 \\
\text { compared with heart failure and carcinoma groups (ANOVA); } \#: p<0.05 \\
\text { compared with heart failure group (Fisher's exact test); } \because: p<0.05 \text { compared } \\
\text { with carcinoma group (Fisher's exact test). }\end{array}$} \\
\hline
\end{tabular}

adenosine deaminase (ADA) levels (which has been proposed as a useful marker for the diagnosis of TB pleural effusions [27, 28]) and high total protein. The exception was one case where there was positive sputum auramine stain and TB culture, with a culture-negative pleural effusion. The decision was made to label these patients as "probable pleural TB" based on these clinical and laboratory criteria rather than on response to antituberculous treatment, since there are a number of other potential factors that could lead to failure of therapy, such as poor compliance and drug-resistant organisms (which are prevalent in the Lima region).

Table 1 summarises the findings on histology, microscopy and culture for the four groups included in the study. Patients in the TB groups were significantly younger than in the control groups (table 2). There was no statistically significant difference in body mass index between TB and congestive heart failure patients. More male than female patients were involved in the study overall (45 males and 19 females). Patients with pleural TB were more likely to be febrile and have pleuritic chest pain than heart failure patients, but otherwise had similar clinical symptoms and signs. PPD testing was performed in 44 cases and four of the controls: this was more likely to be positive ( $\geqslant 10 \mathrm{~mm}$ induration) in patients with definite pleural TB $(p=0.028)$.

Biochemical parameters differed as expected between the groups, with a higher pleural fluid mean total protein and albumin in the TB cases (table 3). Median ADA was over ninefold higher in the TB and probable TB groups than in the heart failure group $(p<0.005)$. The median total leukocyte count in the TB group (predominantly mononuclear cells) was over four-fold greater than in heart failure patients $(p<0.05)$.

\section{MMP concentrations in pleural fluid}

MMP-9 was detectable by zymography at significantly higher levels in the pleural fluid of TB and probable TB patients than in controls (fig. $1 \mathrm{a}$ and $\mathrm{b} ; \mathrm{p}<0.001$ ). The median (interquartile range) in the definite pleural TB group was 105 (60217) $\mathrm{pg} \cdot \mathrm{mL}^{-1}$, in the probable pleural TB group was 57 (44137) $\mathrm{pg} \cdot \mathrm{mL}^{-1}$ and in the control heart failure patients was $<5$ $(0-5) \mathrm{pg} \cdot \mathrm{mL}^{-1}$. In the 10 patients with malignant disease, the pleural fluid MMP-9 concentration was 52 (26-61) $\mathrm{pg} \cdot \mathrm{mL}^{-1}$, significantly lower than in patients with pleural TB $(\mathrm{p}<0.02)$. Representative zymograms also showed that, in contrast to MMP-9, MMP-2 concentrations were similar in all patients. Pleural fluid MMP-1 concentrations did not vary between the groups (fig. 1c).

Pleural MMP-9 concentration did not correlate with any documented clinical parameters but did correlate weakly with ADA concentrations in the pleural fluid $(r=0.37, p<0.005$; Spearman rank).

\section{TIMP-1 and -2 concentrations and MMP ratios in pleural fluid}

Since the matrix-degrading capacity of a cell's secretions depends on a balance between the MMP and TIMP levels, concentrations of TIMP-1 and -2 (the major secreted TIMPs) were measured in the pleural fluid. TIMP-1 concentrations were highest in the pleural TB group (median $5,466 \mathrm{pg} \cdot \mathrm{mL}^{-1}$, interquartile range $\left.(4,394-6,502) \mathrm{pg} \cdot \mathrm{mL}^{-1}\right)$ and significantly

TABLE 3 Biochemical and cellular parameters of pleural fluid from patients and controls

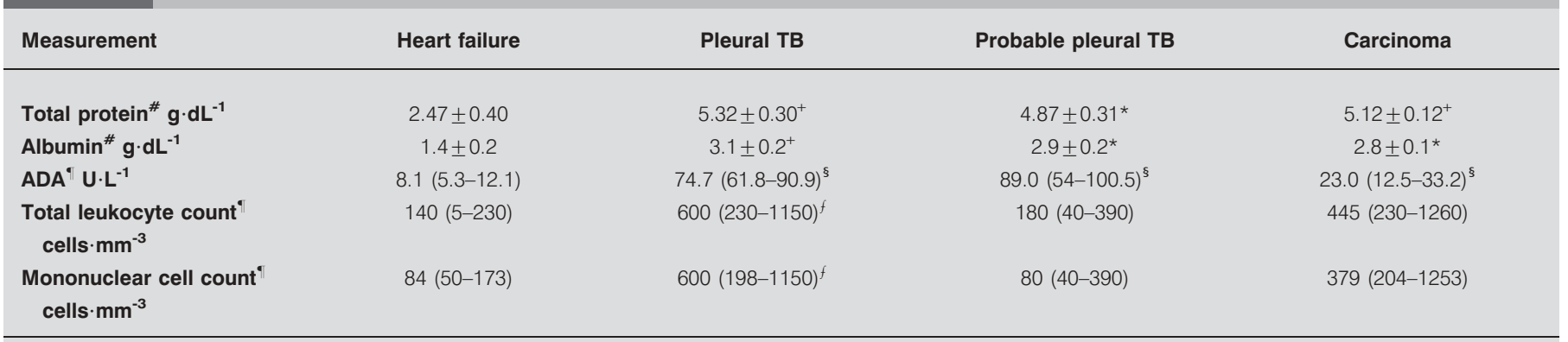

Data are presented as mean \pm SE or median (interquartile range). TB: tuberculosis; ADA: adenosine deaminase. \#: ANOVA with post hoc Tukey honestly significant difference correction; " : Kruskall-Wallis followed by Mann-Whitney test for comparisons of each case group versus control. *: $p<0.05$ versus control; ${ }^{+}: p<0.005$ versus control; ${ }^{\text {s: }} p<0.005$ compared with heart failure and carcinoma groups; ${ }^{f}: p<0.05$ versus heart failure. 


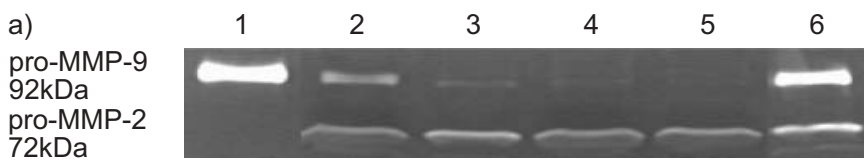

b) 1000
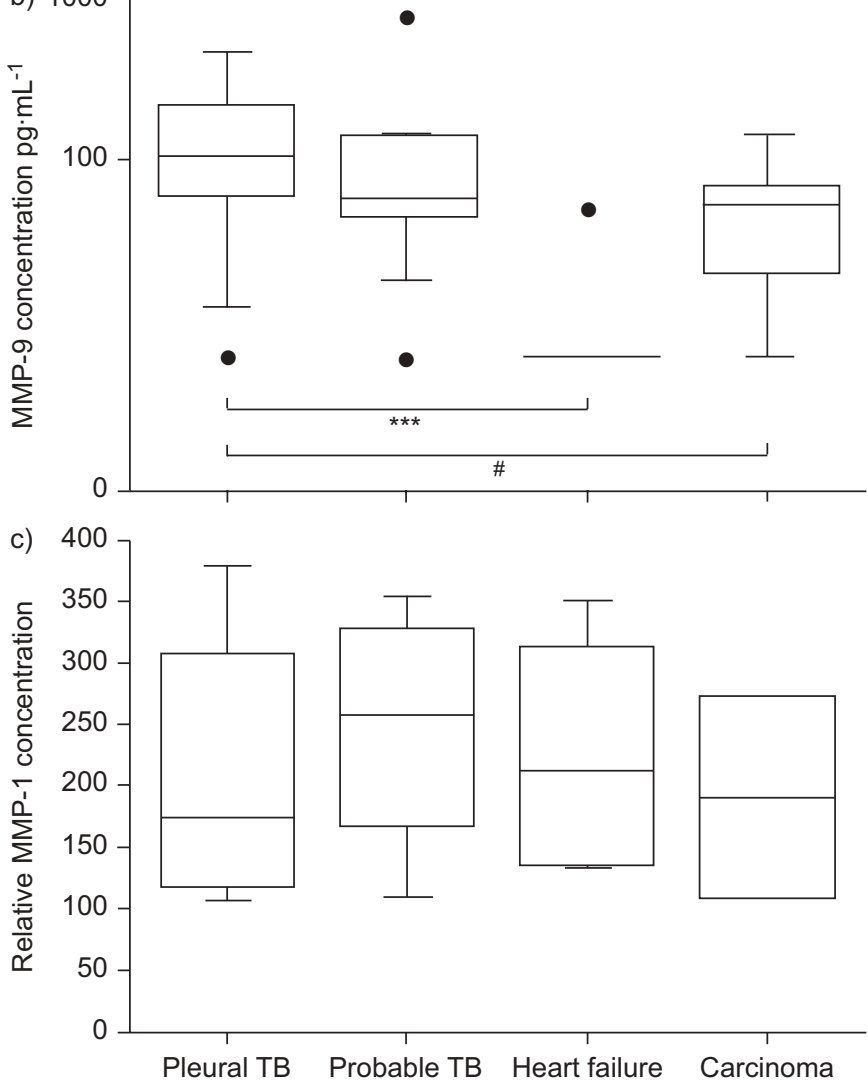

FIGURE 1. a) Representative gelatin zymogram showing the two gelatinolytic bands corresponding to matrix metalloproteinase (MMP)-9 and -2 from six pleural fluid samples. Lane 1: standard aliquot of purified MMP-9; lanes 2, 3 and 6: pleural fluid samples from tuberculosis (TB) cases, lanes 4 and 5 pleural fluid from control cases. b) MMP-9 and c) relative MMP-1 concentrations in pleural fluid from patients with definite pleural TB, probable pleural TB, heart failure and carcinoma. Box-andwhisker plots showing median, interquartile range and $95 \%$ confidence intervals (Cls). - data points falling outside the $95 \%$ Cls. b) ${ }^{* * *}$ : $p<0.001$ (Mann-Whitney); \#: $p<0.02$ (Mann-Whitney). c) No significant differences were observed between the four groups.

greater than in heart failure subjects ( $\mathrm{p}=0.009$; table 4$)$. TIMP-2 concentrations were 20-30-fold less than TIMP-1 concentrations, and did not vary between groups.

The ratio of MMP-9 to TIMP-1 is important in determining net gelatinolytic activity. There was a higher MMP-9/TIMP-1 ratio in TB compared with heart failure patients ( $p=0.004$; fig. 2), suggesting that the increase in TIMP-1 concentration will not compensate for the increase in MMP activity in TB. The MMP-9/TIMP-1 ratio in the group of patients with carcinoma was not significantly different from the TB patients, consistent with the known tendency for matrix degradation in malignant effusions [29].

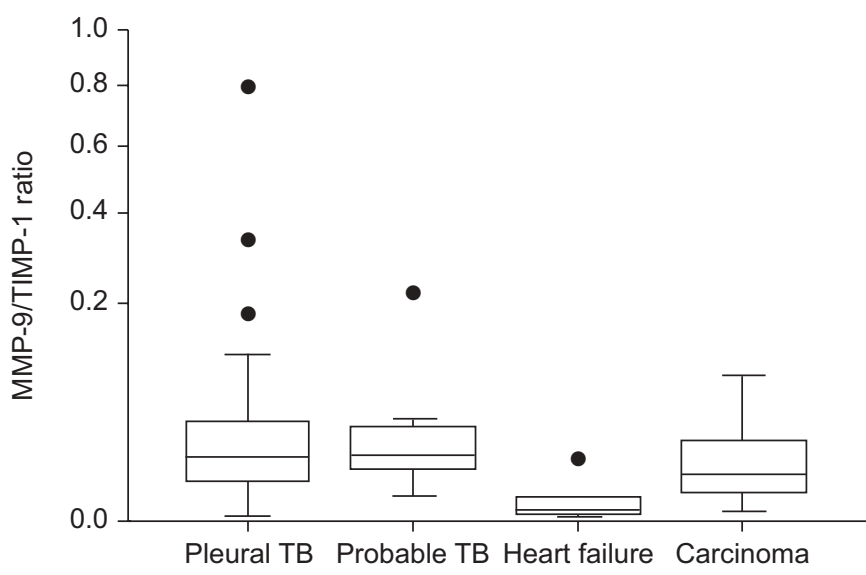

FIGURE 2. Comparison of matrix metalloproteinase (MMP)-9 to tissue inhibitor of metalloproteinase (TIMP)-1 ratio in pleural fluid from patients with definite pleural tuberculosis (TB), probable pleural TB, heart failure and carcinoma. Box-andwhisker plots showing median, interquartile range and 95\% confidence intervals (Cls). : data points falling outside the $95 \% \mathrm{Cls}$. $\mathrm{p}=0.004$ for comparison of the TB groups with the heart failure group.

\section{MMP-9 and TIMP-1 expression in the tuberculous granuloma}

Since MMP-9 expression may be important both in the initial development of the granuloma and in subsequent necrosis, it was next investigated whether granuloma formation was associated with MMP-9 secretion. Median (interquartile range) MMP-9 concentrations were significantly higher in patients in whom granulomas were detected on biopsy, at 108 (61218) $\mathrm{pg} \cdot \mathrm{mL}^{-1}$, compared with $43(12-83) \mathrm{pg} \cdot \mathrm{mL}^{-1}$ in nongranulomatous pleural biopsies ( $p=0.001$; fig. 3 ).

On immunohistochemical analysis of pleural biopsies, there was strong MMP-9 staining in the granuloma at the edge of the caseous necrosis, corresponding to the areas where mononuclear cells are present (fig. 4). MMP-9 staining was less marked towards the periphery of the granuloma. In contrast, TIMP-1 expression in pleural granulomas was at a low level, restricted to a few spindle-shaped cells.

\section{DISCUSSION}

The present study has demonstrated relatively unopposed upregulated MMP-9 activity correlating with granuloma formation in the pleural cavity of patients with tuberculous pleural effusions. MMP-9 concentrations did not relate to clinical symptoms, all of which are common but not exclusive to TB. None of these symptoms are useful markers of disease severity or the extent of inflammatory response within the pleural cavity. In addition, a positive PPD test, which reflects adaptive immunity, did not correlate with MMP-9 expression. This is consistent with the fact that cells of the innate immune response, including monocytes/macrophages, are the most likely source of MMP-9 secretion.

MMP-9 secretion was significantly higher in patients with tuberculous effusions than in patients with malignant pleural disease. At least seven of the 10 malignant effusions in the present study were metastatic in origin. Metastatic effusions are usually characterised by high gelatinolytic expression [30] The higher MMP-9 secretion in TB reflects the remarkable 
TABLE 4 Concentrations of tissue inhibitors of metalloproteinases (TIMPs) in pleural fluid

\begin{tabular}{ccccc} 
& Heart failure & Pleural TB & Probable pleural TB & Carcinoma \\
\hline TIMP-1 ng. $\mathbf{m L}^{-1}$ & $3281(1829-4457)$ & $5466(4394-6502)^{* *}$ & $4665(3540-7216)$ & $4400(2028-6148)$ \\
TIMP-2 $\mathbf{~ g ~} \cdot \mathbf{m L}^{-1}$ & $132(122-135)$ & $122(97-157)$ & $131(121-138)$ & $133(114-157)$ \\
\hline
\end{tabular}

Data are presented as median (interquartile range). ${ }^{*}: p<0.01$ compared with control (heart failure) group.

capacity of $M$. tuberculosis to induce an extensive inflammatory response in the human host, and may reflect a particular involvement of MMP-9 in granuloma formation in response to this organism.

M. tuberculosis infection of monocytes and macrophages induces MMP-9 secretion [9, 31-33], and direct infection of these cells in vivo with TB is likely to be a major source of MMP-9 secretion. However, cytokine networks may be a further powerful stimulus to MMP-9 induction in tuberculous pleurisy. Mononuclear cells infected with $M$. tuberculosis secrete tumour necrosis factor (TNF)- $\alpha$ and IL-1 $\beta$, which are capable of driving MMP-9 secretion from uninfected monocytes [10] and stromal cells, including mesothelial cells, endothelial cells and fibroblasts [34-36]. TNF- $\alpha$ is required for granuloma formation in TB, and anti-TNF therapy is associated with granuloma breakdown and dissemination of TB [37]. This may, in part, reflect the requirement for TNF- $\alpha-$ dependent MMP-9 secretion in granuloma formation. The current authors found that pleural MMP-9 concentration correlated with granuloma formation, and cells adjacent to caseous necrosis in the granuloma expressed high-level MMP-9, possibly implicating MMP-9 as a component in the process of caseation. It is unclear from the present study whether MMP-9 secretion is a deleterious consequence of granuloma formation or itself is required for granuloma formation. A recent murine study has implicated MMP-9 in pulmonary tuberculous granuloma formation [38], suggesting

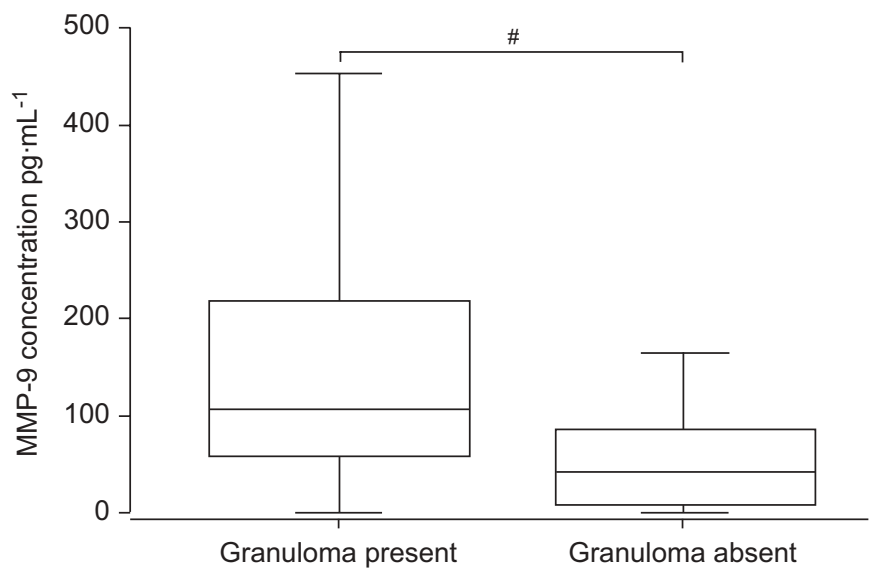

FIGURE 3. Comparison of matrix metalloproteinase (MMP)-9 concentrations in pleural fluid in the presence or absence of granulomas on pleural biopsy. Box-andwhisker plots showing median, interquartile range and $95 \%$ confidence intervals. \#: $\mathrm{p}=0.001$. a protective role for MMP-9. However, the structure and regulation of tuberculous granulomas in mice is very different and extrapolation of murine data to humans is difficult.

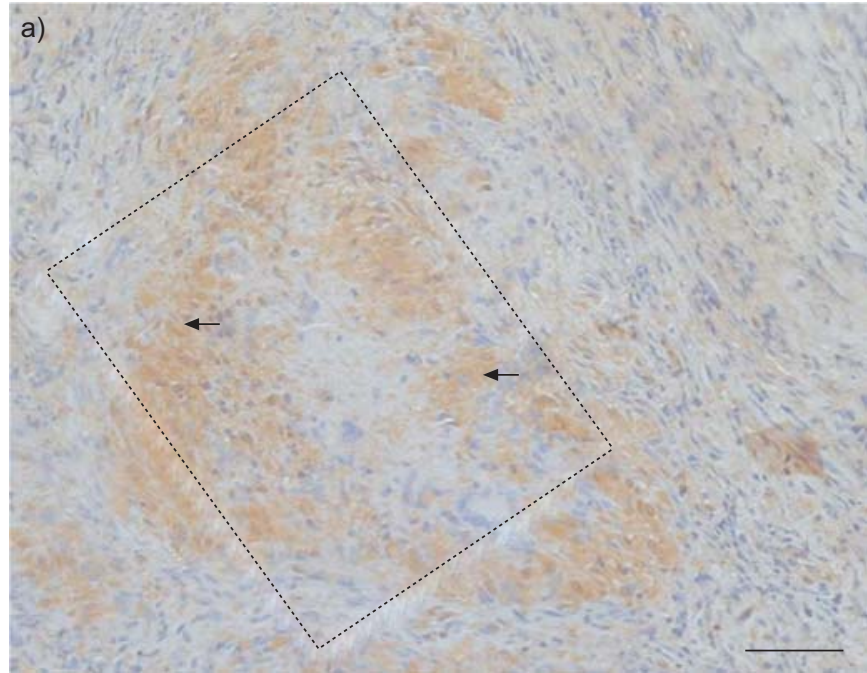

b)

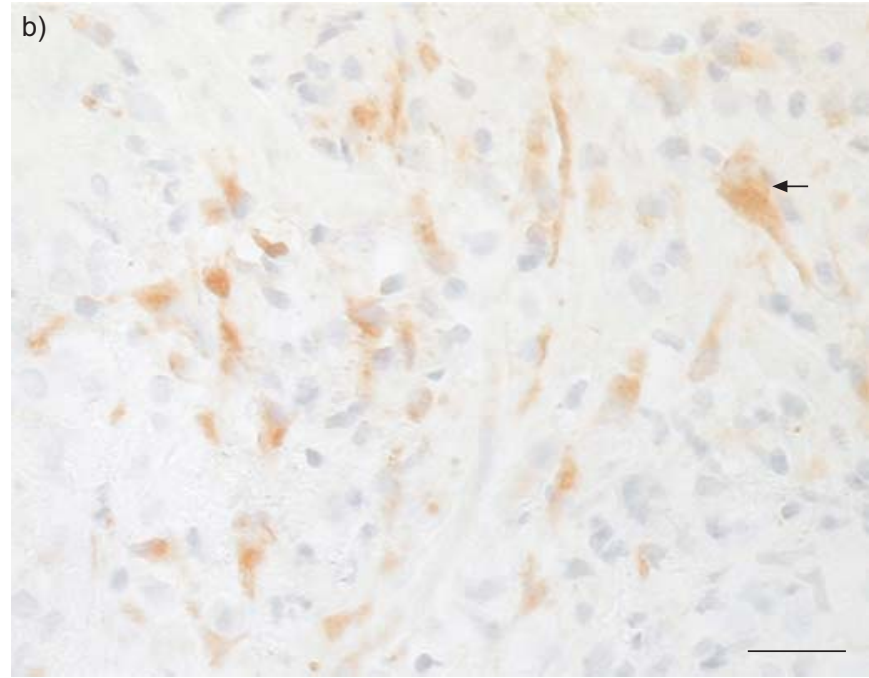

FIGURE 4. a) Matrix metalloproteinase (MMP)-9 immunostaining in a granuloma. Pleural biopsy granulomatous material from patients with culture-positive tuberculosis was immunostained for MMP-9. The centre of the granuloma is highlighted $(\cdots \cdots)$ MMP-9 is seen as brown staining against a blue background (arrows). MMP-9 is most marked in the central region of the granuloma near the region of caseous necrosis and becomes more scant towards the granuloma periphery. b) Immunostaining for tissue inhibitor of metalloproteinase (TIMP)-1. The same samples were stained for TIMP-1, again labelled brown against a blue background (arrow). Granulomas stained relatively weakly for TIMP-1 and staining was restricted to spindle-shaped fibroblastlike cells. a) Scale bar $=80 \mu \mathrm{m}$, b) scale bar $=20 \mu \mathrm{m}$. 
There are several other important potential consequences of relatively unopposed MMP-9 activity in the pleural cavity. Pleural MMP-9 will facilitate the degradation of basement membrane of the mesothelial layer and pleural blood vessels by proteolysis of type IV collagen. This will contribute to accumulation of fluid within the pleural space and aid migration of leukocytes from the circulation. In addition, MMP-9 will cleave and activate CXC chemokine ligand 8 [5], a potent chemoattractant that is important in recruitment of mononuclear cells in TB pleurisy [39], thereby increasing the inflammatory response.

Activated macrophages are known to secrete MMP-1, and stromal cells can secrete MMP-1 in response to cytokines such as TNF- $\alpha$ or IL-1 $\beta$. Therefore, the absence of upregulation of MMP-1 in the pleural fluid in TB patients was unexpected, although it supports the hypothesis that matrix degradation in pleural TB is MMP-9 driven. Additionally, the uniform secretion of MMP-2 in the pleural fluid in all patient groups in the present study is consistent with a previous study investigating pleural effusions of transudative (congestive heart failure), parainfectious and paraneoplastic origin [15] and may suggest that MMP-2 has a role in homeostasis rather than inflammation in the pleural space.

The specific and marked upregulation of MMP-9 in the TB effusions raises the question of whether a specific threshold concentration of this enzyme can discriminate between tuberculous and other exudative effusions, including neoplastic or parapneumonic. It has previously been shown that CSF MMP-9 concentrations are higher in tuberculosis than bacterial meningitis [10], but the current study does not include any patients with a clinical or laboratory diagnosis of parapneumonic effusions or empyema to allow this to be investigated for pleural infection. Additionally, the number of subjects in the present study with malignant effusions was small and the subjects were older than those with tuberculosis, such that attempts to determine whether MMP-9 concentrations can distinguish malignant and tuberculous effusions are statistically unsafe. Further investigation of MMP-9 in parapneumonic and malignant effusions compared with tuberculous effusions is required and will be the subject of a further adequately powered study.

TIMP-1 was present in concentrations of at least 20-30-fold that of TIMP-2 and is, therefore, likely to be the functionally more important TIMP in pleural TB. The low expression of TIMP-1 within the granuloma suggests that there is relatively unopposed gelatinolytic activity within this structure, with a consequent overall tendency for matrix degradation. Although TIMP-1 was elevated in pleural fluid in TB patients, the MMP-9/TIMP-1 ratio indicates that even this TIMP secretion is unlikely to be functionally sufficient to control matrix-degrading enzymatic activity.

In summary, the present study has demonstrated high and functionally unopposed matrix metalloproteinase-9 activity in pleural tuberculosis correlating with the presence of granulomas. The data suggest that unopposed matrix metalloproteinase- 9 activity is crucial to the pathogenesis of tuberculous pleurisy.

\section{ACKNOWLEDGEMENTS}

The authors would like to thank the residents of the Dept of Medicine, Hospital Arzobispo Loayza and the attending staff of the Dept of Pulmonology, Dos De Mayo Hospital (both Lima, Peru) for their help with this study.

\section{REFERENCES}

1 Valdés L, Alvarez D, San José E, et al. Tuberculous pleurisy: a study of 254 patients. Arch Intern Med 1998; 158: 2017-2021.

2 Mohammed KA, Nasreen N, Ward MJ, Mubarak KK, Rodriguez-Panadero F, Antony VB. Mycobacteriummediated chemokine expression in pleural mesothelial cells: role of $\mathrm{C}-\mathrm{C}$ chemokines in tuberculous pleurisy. J Infect Dis 1998; 178: 1450-1456.

3 Visse R, Nagase H. Matrix metalloproteinases and tissue inhibitors of metalloproteinases: structure, function, and biochemistry. Circ Res 2003; 92: 827-839.

4 Schönbeck U, Mach F, Libby P. Generation of biologically active IL-1 $\beta$ by matrix metalloproteinases: a novel caspase-1independent pathway of IL-1 $\beta$ processing. J Immunol 1998; 161: 3340-3346.

5 Van den Steen PE, Wuyts A, Husson SJ, Proost P, Van Damme J, Opdenakker G. Gelatinase B/MMP-9 and neutrophil collagenase/MMP-8 process the chemokines human GCP-2/CXCL6, ENA-78/CXCL5 and mouse GCP-2/LIX and modulate their physiological activities. Eur J Biochem 2003; 270: 3739-3749.

6 Lark MW, Bayne EK, Flanagan J, et al. Aggrecan degradation in human cartilage. Evidence for both matrix metalloproteinase and aggrecanase activity in normal, osteoarthritic, and rheumatoid joints. J Clin Invest 1997; 100: 93-106.

7 Atkinson JJ, Senior RM. Matrix metalloproteinase-9 in lung remodeling. Am J Respir Cell Mol Biol 2003; 28: 12-24.

8 Hautamaki RD, Kobayashi DK, Senior RM, Shapiro SD. Requirement for macrophage elastase for cigarette smokeinduced emphysema in mice. Science 1997; 277: 2002-2004.

9 Quiding-Järbrink M, Smith DA, Bancroft GJ. Production of matrix metalloproteinases in response to mycobacterial infection. Infect Immun 2001; 69: 5661-5670.

10 Price NM, Farrar J, Tran TT, Nguyen TH, Tran TH, Friedland JS. Identification of a matrix-degrading phenotype in human tuberculosis in vitro and in vivo. J Immunol 2001; 166: 4223-4230.

11 Chang JC, Wysocki A, Tchou-Wong KM, Moskowitz N, Zhang Y, Rom WN. Effect of Mycobacterium tuberculosis and its components on macrophages and the release of matrix metalloproteinases. Thorax 1996; 51: 306-311.

12 Price NM, Gilman RH, Uddin J, Recavarren S, Friedland JS. Unopposed matrix metalloproteinase-9 expression in human tuberculous granuloma and the role of TNF- $\alpha$-dependent monocyte networks. J Immunol 2003; 171: 5579-5586.

13 Harris JE, Nuttall RK, Elkington PT, et al. Monocyteastrocyte networks regulate matrix metalloproteinase gene expression and secretion in central nervous system tuberculosis in vitro and in vivo. J Immunol 2007; 178: 1199-1207.

14 Brinckerhoff CE, Matrisian LM. Matrix metalloproteinases: a tail of a frog that became a prince. Nat Rev Mol Cell Biol 2002; 3: 207-214.

15 Eickelberg O, Sommerfeld CO, Wyser C, et al. MMP and TIMP expression pattern in pleural effusions of different origins. Am J Respir Crit Care Med 1997; 156: 1987-1992. 
16 Hoheisel G, Sack U, Hui DS, et al. [Immunohistochemical localization of matrix metalloproteinases (MMP) and tissue inhibitors of metalloproteinases (TIMP) in tuberculous pleuritis]. Pneumologie 2004; 58: 305-308.

17 Iglesias D, Alegre J, Alemán C, et al. Metalloproteinases and tissue inhibitors of metalloproteinases in exudative pleural effusions. Eur Respir J 2005; 25: 104-109.

18 Park KJ, Hwang SC, Sheen SS, Oh YJ, Han JH, Lee KB. Expression of matrix metalloproteinase-9 in pleural effusions of tuberculosis and lung cancer. Respiration 2005; 72: 166-175.

19 St-Pierre Y, Van Themsche C, Estève PO. Emerging features in the regulation of MMP-9 gene expression for the development of novel molecular targets and therapeutic strategies. Curr Drug Targets Inflamm Allergy 2003; 2: 206-215.

20 Ma C, Tarnuzzer RW, Chegini N. Expression of matrix metalloproteinases and tissue inhibitor of matrix metalloproteinases in mesothelial cells and their regulation by transforming growth factor- $\beta 1$. Wound Repair Regen 1999; 7: 477-485.

21 Giacomini E, Iona E, Ferroni L, et al. Infection of human macrophages and dendritic cells with Mycobacterium tuberculosis induces a differential cytokine gene expression that modulates T cell response. J Immunol 2001; 166: 70337041.

22 Idell S, Girard W, Koenig KB, McLarty J, Fair DS. Abnormalities of pathways of fibrin turnover in the human pleural space. Am Rev Respir Dis 1991; 144: 187-194.

23 Bachmeier BE, Iancu CM, Jochum M, Nerlich AG. Matrix metalloproteinases in cancer: comparison of known and novel aspects of their inhibition as a therapeutic approach. Expert Rev Anticancer Ther 2005; 5: 149-163.

24 Jackson C, Nguyen M, Arkell J, Sambrook P. Selective matrix metalloproteinase (MMP) inhibition in rheumatoid arthritis - targetting gelatinase A activation. Inflamm Res 2001; 50: 183-186.

25 Villarreal FJ, Griffin M, Omens J, Dillmann W, Nguyen J, Covell J. Early short-term treatment with doxycycline modulates postinfarction left ventricular remodeling. Circulation 2003; 108: 1487-1492.

26 Hurewitz AN, Zucker S, Mancuso P, et al. Human pleural effusions are rich in matrix metalloproteinases. Chest 1992; 102: 1808-1814.

27 Burgess LJ, Reuter H, Carstens ME, Taljaard JJ, Doubell AF. The use of adenosine deaminase and interferon- $\gamma$ as diagnostic tools for tuberculous pericarditis. Chest 2002; 122: 900-905.
28 Greco S, Girardi E, Masciangelo R, Capoccetta GB Saltini C. Adenosine deaminase and interferon- $\gamma$ measurements for the diagnosis of tuberculous pleurisy: a metaanalysis. Int J Tuberc Lung Dis 2003; 7: 777-786.

29 van Hensbergen Y, Broxterman HJ, Hanemaaijer R, et al. Soluble aminopeptidase N/CD13 in malignant and nonmalignant effusions and intratumoral fluid. Clin Cancer Res 2002; 8: 3747-3754.

30 Macaulay VM, O'Byrne KJ, Saunders MP, et al. Phase I study of intrapleural batimastat (BB-94), a matrix metalloproteinase inhibitor, in the treatment of malignant pleural effusions. Clin Cancer Res 1999; 5: 513-520.

31 Ragno S, Romano M, Howell S, Pappin DJ, Jenner PJ, Colston MJ. Changes in gene expression in macrophages infected with Mycobacterium tuberculosis: a combined transcriptomic and proteomic approach. Immunology 2001; 104: 99-108.

32 Rivera-Marrero CA, Schuyler W, Roser S, Roman J. Induction of MMP-9 mediated gelatinolytic activity in human monocytic cells by cell wall components of Mycobacterium tuberculosis. Microb Pathog 2000; 29: 231-244.

33 Rivera-Marrero CA, Schuyler W, Roser S, Ritzenthaler JD, Newburn SA, Roman JM. M. tuberculosis induction of matrix metalloproteinase-9: the role of mannose and receptor-mediated mechanisms. Am J Physiol Lung Cell Mol Physiol 2002; 282: L546-L555.

34 Nakamura Y, Esnault S, Maeda T, Kelly EA, Malter JS, Jarjour NN. Ets-1 regulates TNF- $\alpha$-induced matrix metalloproteinase- 9 and tenascin expression in primary bronchial fibroblasts. J Immunol 2004; 172: 1945-1952.

35 Martin J, Yung S, Robson RL, Steadman R, Davies M. Production and regulation of matrix metalloproteinases and their inhibitors by human peritoneal mesothelial cells. Perit Dial Int 2000; 20: 524-533.

36 Zhu Z, Yao J, Wang F, Xu Q. TNF- $\alpha$ and the phenotypic transformation of human peritoneal mesothelial cell. Chin Med J (Engl) 2002; 115: 513-517.

37 Keane J, Gershon S, Wise RP, et al. Tuberculosis associated with infliximab, a tumor necrosis factor- $\alpha$-neutralizing agent. N Engl J Med 2001; 345: 1098-1104.

38 Taylor JL, Hattle JM, Dreitz SA, et al. Role for matrix metalloproteinase 9 in granuloma formation during pulmonary Mycobacterium tuberculosis infection. Infect Immun 2006; 74: 6135-6144.

39 Pace E, Gjomarkaj M, Melis M, et al. Interleukin-8 induces lymphocyte chemotaxis into the pleural space. Role of pleural macrophages. Am J Respir Crit Care Med 1999; 159: 1592-1599. 